\title{
Lung endothelial monocyte-activating protein 2 is a mediator of cigarette smoke-induced emphysema in mice
}

\author{
Matthias Clauss, ${ }^{1}$ Robert Voswinckel, ${ }^{2}$ Gangaraju Rajashekhar, ${ }^{1}$ Ninotchka L. Sigua, ${ }^{3}$ \\ Heinz Fehrenbach, ${ }^{4}$ Natalia I. Rush, ${ }^{3}$ Kelly S. Schweitzer, ${ }^{3}$ Ali Ö. Yildirim, ${ }^{5}$ Krzysztof Kamocki, ${ }^{3}$ \\ Amanda J. Fisher, ${ }^{3,6}$ Yuan Gu, ${ }^{3}$ Bilal Safadi, ${ }^{3}$ Sandeep Nikam, ${ }^{2}$ Walter C. Hubbard, ${ }^{7}$ \\ Rubin M. Tuder, ${ }^{8}$ Homer L. Twigg III, ${ }^{3}$ Robert G. Presson, ${ }^{3,6}$ Sanjay Sethi, ${ }^{9}$ and Irina Petrache ${ }^{3,10}$ \\ ${ }^{1}$ Indiana Center for Vascular Biology and Medicine and Department of Cellular and Integrative Physiology, Indiana University, Indianapolis, Indiana, USA. \\ 2Max-Planck-Institute for Heart and Lung Research, Bad Nauheim, Germany. ${ }^{3}$ Department of Medicine and Center for Immunobiology, Indiana University, \\ Indianapolis, Indiana, USA. ${ }^{4}$ Research Center Borstel, Leibniz-Center for Medicine and Biosciences, Borstel, Germany. ${ }^{5}$ Institute of Lung Biology and Disease, \\ German Research Center for Environmental Health, Helmholtz Zentrum, Munich, Germany. ${ }^{6}$ Department of Anesthesia, Indiana University, Indianapolis, \\ Indiana, USA. 'Department of Pharmacology, The Johns Hopkins University, Baltimore, Maryland, USA. ${ }^{8}$ Department of Medicine, \\ University of Colorado Health Science Center, Denver, Colorado, USA. PDepartment of Medicine, New York University School of Medicine, \\ Buffalo, New York, USA. ${ }^{10}$ Richard L. Roudebush Veteran Affairs Medical Center, Indianapolis, Indiana, USA.
}

\begin{abstract}
Pulmonary emphysema is a disease characterized by alveolar cellular loss and inflammation. Recently, excessive apoptosis of structural alveolar cells has emerged as a major mechanism in the development of emphysema. Here, we investigated the proapoptotic and monocyte chemoattractant cytokine endothelial monocyte-activating protein 2 (EMAPII). Lung-specific overexpression of EMAPII in mice caused simplification of alveolar structures, apoptosis, and macrophage accumulation, compared with that in control transgenic mice. Additionally, in a mouse model of cigarette smoke-induced (CS-induced) emphysema, EMAPII levels were significantly increased in murine lungs. This upregulation was necessary for emphysema development, as neutralizing antibodies to EMAPII resulted in reduced alveolar cell apoptosis, inflammation, and emphysema-associated structural changes in alveoli and small airways and improved lung function. The mechanism of EMAPII upregulation involved an apoptosis-dependent feed-forward loop, since caspase-3 instillation in the lung markedly increased EMAPII expression, while caspase inhibition decreased its production, even in transgenic EMAPII mice. These findings may have clinical significance, as both current smokers and exsmoker chronic obstructive pulmonary disease (COPD) patients had increased levels of secreted EMAPII in the bronchoalveolar lavage fluid compared with that of nonsmokers. In conclusion, we suggest that EMAPII perpetuates the mechanism of CS-induced lung emphysema in mice and, given its secretory nature, is a suitable target for neutralization antibody therapy.
\end{abstract}

\section{Introduction}

Pulmonary emphysema is characterized by loss of both matrix and cellular elements of the lung, thus impairing gas exchange between the alveolar space and the capillary blood. Over the past decades, inflammation and a protease/antiprotease imbalance have been the focus of studies that addressed lung destruction following chronic cigarette smoking. More recently, excessive apoptosis of structural alveolar cells has emerged as a major mechanism contributing to the development of lung emphysema, accounting for the unique nature of lung destruction as compared with that with other inflammatory lung diseases (1-3). The identification of molecular mediators that trigger and integrate lung responses involving inflammation and alveolar cell apoptosis in response to cigarette smoke (CS) exposure offers the potential for development of novel therapies.

Authorship note: Matthias Clauss and Robert Voswinckel contributed equally to this work.

Conflict of interest: Matthias Clauss, Robert Voswinckel, and Irina Petrache have filed a patent application for the discovery of EMAPII as a therapeutic target in emphysema and for its potential use as a biomarker for the disease.

Citation for this article: J Clin Invest. 2011;121(6):2470-2479. doi:10.1172/JCI43881
Endothelial monocyte-activating protein 2 (EMAPII) was initially discovered as a proinflammatory endothelial- and monocyte-activating polypeptide $(4,5)$ and later identified as an antangiogenic molecule, which specifically induces apoptosis in endothelial cells $(6,7)$. This property of EMAPII may be highly relevant to emphysema, because lung endothelial cell apoptosis was sufficient to recapitulate key pathological features of this disease (3). The monocyte-activating and chemotactic activities of EMAPII share homology with IL-8 within the consensus sequences for chemokines. In addition, EMAPII uses the CXCR3 receptor (8), which also binds the chemokines IP-10/CXCL10, I-TAC/CXCL11, MIG/CXCL9, and PF4/CXCL4, leading to the classification of EMAPII as a nonclassical chemokine (8-10). EMAPII is expressed in the cytosol of all cell types as part of the tRNA-synthetase multienzyme complex (11) and is upregulated by general cellular stress, hypoxia, and LPS (12-15). EMAPII is secreted from cells as a $43-\mathrm{kDa}$ proform or as 23 - and $28-\mathrm{kDa}$ mature forms generated proteolytically by either intracellular (caspase-3, caspase-7) or extracellular proteinases (MMP-9, neutrophil elastase, and cathepsin) (16-20), many of which are known to participate in the chronic obstructive pulmonary disease (COPD) lung pathobiology (21). 
Using conditional lung overexpression of EMAPII and a neutralizing antibody to EMAPII in a mouse model of CS-induced emphysema, we provide functional data that implicate EMAPII as a mediator of CS-induced lung injury in COPD. Based on the evidence presented here that EMAPII is both inducing cell death in alveoli of the lung as well as being produced in an apoptosis-dependent manner, we suggest EMAPII is an amplifying mechanism of CSinduced lung emphysema and, given its secretory nature, a suitable target for neutralization antibody therapy.

\section{Results}

Lung-specific EMAPII overexpression induces alveolar apoptosis and emphysema-like pathology of the lung. To test the hypothesis that EMAPII upregulation in the lung is sufficient to induce emphysema-like changes, we generated a mouse with tetracycline/doxycycline-inducible lung-specific overexpression of EMAPII, using a transactivator driven by Clara cell-specific protein (CCSP) promoter elements, previously shown to induce transgenic expression in both Clara and alveolar type 2 cells $(22,23)$. The doxycyclineinduced EMAPII lung overexpression was initiated at the earliest at 9 weeks of age, when mouse lung maturation is complete. Because only the double-transgenic (DT) EMAPII/CSSP transactivator but not the single-transgenic (ST) mice are expected to produce EMAPII upon induction with doxycycline, both CCSP transactivator background effects and doxycycline effects were included within the control groups. As expected, doxycycline administration led to increased lung EMAPII secretion in the lungs, measured in the bronchoalveolar lavage fluid (BALF) and lung parenchyma of 3 independent DT lines, but not in control ST littermates, starting as early as 24 hours (Figure 1A; schematic in top panel of Figure 1B). By immunohistochemistry with EMAPII antiserum, lung EMAPII expression was markedly enhanced in the lung parenchyma (bottom panel of Figure 1B), in which it colocalized with the epithelial cell marker pro-SPC (Supplemental Figure 1; supplemental material available online with this article; doi:10.1172/JCI43881DS1) but was absent in other tissues, such as the liver and heart (Supplemental Figure 1). Overexpressing EMAPII in DT mice (EMAPII/ CCSP) in comparison to ST control mice (CCSP-only) induced a 3 -fold increase in caspase-3 activity in the lungs, as early as 4 weeks after EMAPII induction (Figure 1C). Immunofluorescence staining revealed alveolar localization of activated caspase-3-positive cells, while confocal microscopy after double staining with VE-cadherin indicated that the proapoptotic effects of EMAPII involved alveolar endothelial cells (Supplemental Figure 1). In addition, lung lysates from EMAPII-overexpressing DT mice exhibited a marked increase in the numbers of alveolar macrophages in the BALF (Figure 1D) and lung parenchyma, detected by immunohistochemistry (Supplemental Figure 1) after induction with doxycycline for 28 weeks. Furthermore, a prolonged EMAPII overexpression (for 28 weeks) in DT mice increased the size of acinar airspaces (comprising alveoli and alveolar ducts) visualized on H\&E-stained sections of paraffin-embedded and Richardson-stained glycol methacrylateembedded lung sections (Figure 1E, bottom panels), when compared with that of ST controls, which do not express EMAPII upon doxycycline treatment (Figure 1E). These changes were reflected by significant increases in the mean linear intercept (MLI) measurements (Figure 1F) and in the alveolar volume stereotactic measurements (Supplemental Figure 1). These data demonstrate that postdevelopmental EMAPII upregulation in the lung is by itself sufficient to trigger features of emphysema.
Neutralization of EMAPII levels markedly reduces CS-induced lung emphysema in mice. Based on previous findings that EMAPII is produced and released by apoptosis, hypoxia $(12,24)$, and cellular stress (13), we investigated whether EMAPII is induced in the lung in vivo upon exposure to CS. We measured EMAPII protein expression in the DBA/ 2 mouse strain, which develops emphysema after chronic exposure to CS as early 16 weeks, exhibiting a $20 \%$ increase in airspace size, compared with only a $9 \%$ increase measured in the C57BL/6 strain at this time point, respectively (25). CS exposure for only 4 weeks significantly increased the 2 forms of EMAPII expression in the lung parenchyma of DBA/ 2 mice, measured by immunoblotting (Figure 2A).

Next, we addressed the cellular localization of EMAPII expression in normal and CS-exposed mice. Under ambient air conditions, lungs of control mice showed sparse EMAPII expression that colocalized mostly with CD11b-labeled alveolar macrophages (Figure 2B). In contrast, cigarette smoking robustly increased both intracellular and extracellular EMAPII production, which colocalized with both macrophages and alveolar septal cells (Figure 2B).

To functionally assess the role of the secreted EMAPII in CSinduced lung injury and emphysema, we used specific EMAPII neutralizing monoclonal antibodies, which we demonstrated to completely neutralize EMAPII-induced apoptosis in cultured lung microvascular endothelial cells (Supplemental Figure 2 and ref. 26). Antibodies $(50 \mu \mathrm{g} /$ application) were administered directly to the lung via inhalation of a nebulized solution, which showed effective deposition in the lung parenchyma at 15 minutes by fluorescence microscopy of the lung and at 4 hours by immune adsorption analysis of recovered biotinylated antibody from plasma (Supplemental Figure 3). This method of administration has the advantages of targeting the local EMAPII pool and has been previously shown to allow the use of lower antibody doses compared with the systemic route (27). The timing of antibody delivery was chosen to follow the increases in EMAPII detected in response to CS exposure, while the duration of antibody treatment was limited to 4 weeks to minimize or avoid nonspecific immunological side effects. DBA $/ 2$ mice were first exposed to CS alone for 8 weeks, followed by targeting EMAPII with neutralizing antibodies between weeks 9 to 12 and 4 additional weeks of CS exposure (treatment protocol depicted in Figure 2C). The administration of EMAPII-neutralizing antibody significantly decreased lung apoptosis measured by caspase- 3 activity in tissue lysates (Figure $2 \mathrm{D})$, by decreased expression of active caspase- 3 in cells in lung parenchyma (Supplemental Figure 3) and by measurement of ceramide 16:0 in lung homogenates (Supplemental Figure 3). In addition, this treatment decreased the number of inflammatory cells retrieved in the BALF (Figure 2E), particularly alveolar macrophages and neutrophils (Supplemental Figure 3), and reduced the number of neutrophils in the lung parenchyma (Supplemental Figure 3). Furthermore, anti-EMAPII antibodies significantly improved the lung static compliance (Figure 2F) by almost $40 \%$. Importantly, consistent with these functional data, neutralization of EMAPII abolished the CS-induced airspace enlargement measured as a $19.4 \%$ increase in MLI compared with that in airexposed mice, which is in a typical range for CS-induced emphysema mouse models (Figure 2, G and $\mathrm{H}$ ). Interestingly, neutralizing EMAPII antibodies had no effect on CS-induced large airway epithelial remodeling but restored the thickness of the epithelial layer of small airways (smaller than $150 \mu \mathrm{m}$ in diameter), which was significantly reduced by CS exposure (Supplemental Figure 3). 

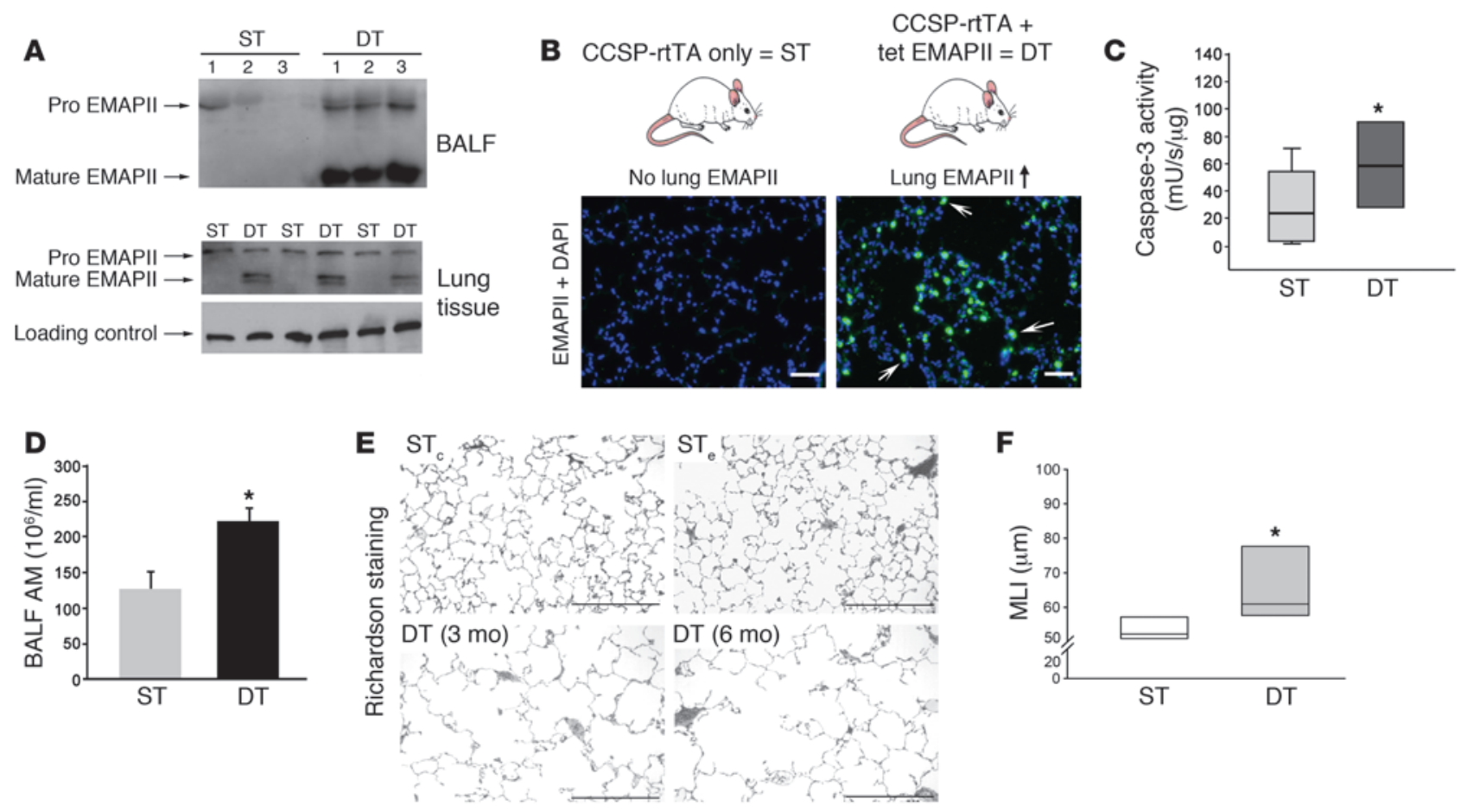

\section{Figure 1}

Lung-specific EMAPII overexpression induces emphysema-like changes. (A) EMAPII detected by Western blot in BALF (24 hours) and lung lysates after induction with doxycycline (3 weeks); vinculin was used as loading control. Lanes 1, 2, and 3 represent independent lines from either ST (CCSP-rtTA) or DT (EMAPII/CCSP-rtTA) mice. (B) Schematic of transgenic induction (top). EMAPII expression detected in lung cryosections by specific EMAPII polyclonal antiserum immunostaining (green) and DAPI (nuclei, blue) after transgenic induction in ST versus DT mice (3 weeks) (bottom). Note marked alveolar EMAPII staining (arrows) in the DT mice. Scale bar: 50 um. (C) Caspase-3 activity in lung lysates of ST or DT mice (4 weeks of induction; ${ }^{*} P<0.05$, ANOVA). (D) Macrophage counts in the BALF of ST mice or DT mice (6 months; mean + SEM; ${ }^{\star} P<0.05$, Student's $t$ test). AM, alveolar macrophage. (E) Representative photomicrographs of Richardson-stained glycol methacrylate lung sections (scale bar: $300 \mu \mathrm{m}$ ) of non-EMAPII-expressing ST CCSP-rtTA (STc) or ST EMAPII (STe) mice compared with EMAPII/CCSP-rtTA mice (DT) after 3 and 6 months of induction, respectively. Note the increase of airspace diameters, consistent with emphysema-like simplification of alveolar structures in DT mice but not in control mice. (F) Morphometry of H\&E-stained lungs from ST and DT mice induced for 6 months. Acinar airspace size was expressed as MLI ( ${ }^{*} P=0.01$, ANOVA; $n=5-6$ at 3 months; $n=7-8$ at 6 months). (C and $\left.\mathbf{F}\right)$ Lines within the boxes show medians; bounds of the boxes show 25th and the 75th percentiles of the data, respectively; and whiskers show outliers, if applicable (5th and 95th percentiles, respectively).

Together, these data demonstrate that treatment with neutralizing antibodies to EMAPII reduced pathological changes in both alveoli and small airways and reduced emphysema development even after a considerable period of CS exposure.

A feed-forward loop linking lung apoptosis and EMAPII. Because EMAPII is released in response to apoptotic stimuli and by itself triggers endothelial apoptosis, we addressed the hypothesis that EMAPII enhances its own expression in an apoptosis-dependent manner, forming a feed-forward loop in the lung. First, we used 2 established approaches of apoptosis induction in the lung parenchyma, i.e., blockade of the VEGF receptor, which induces endothelial cell apoptosis and caspase-3 activation (28) and intratracheal (i.t.) application of active caspase-3, combined with a transfection reagent for intracellular access $(29,30)$. Treatment with the VEGF receptor inhibitor SU5416 markedly increased pro-EMAPII and, to a lesser extent, mature EMAPII in the lung, as assessed by Western blotting (Figure 3, A and B). The measurements of EMAPII expression as arbitrary units reflect normalization of density of Western blot EMAPII signals to those of housekeeping proteins and therefore reflect relative expression of EMAPII from each independent experiment and cannot be directly compared with arbitrary units of EMAPII expression among other experiments, mouse strains, or tissues. Similarly, i.t. active caspase-3 instillations significantly increased EMAPII expression in the lung (Figure 3C). Of note, both pro- and mature EMAPII manifested similar proapoptotic properties, as shown by TUNEL assay in primary human lung microvascular endothelial cells (Supplemental Figure 2). These effects were recapitulated in vivo, where direct lung delivery via aerosol nebulization of recombinant mature EMAPII protein 3 times weekly for 2 weeks $(5 \mu \mathrm{g} / \mathrm{ml})$ activated caspase- 3 in lung lysates (Figure 3D). This effect was dose dependent and was associated with airspace simplification after 4 weeks of administration (Supplemental Figure 4), measured as a decrease in surface-to-volume ratio by stereological morphometry, indicative of emphysema development (31, 32). The EMAPII-induced caspase activation was inhibited by concomitant application of a general caspase inhibitor quinoline-ValAsp-difluorophenoxymethylketone (Q-VD-OPH) (Figure 3D), a new generation inhibitor with reduced toxicity and increased specificity in comparison with other halogenated methylketone-based caspase inhibitors (33). These results suggest that EMAPII is upregulated 
A

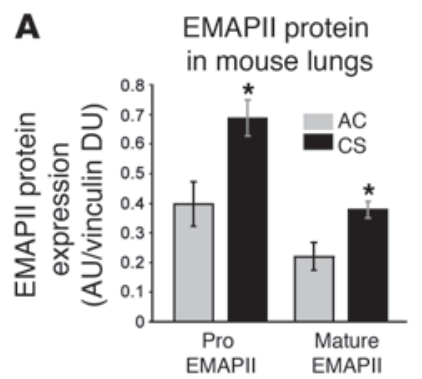

B

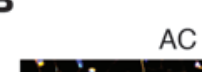

EMAPII localization in mouse lungs
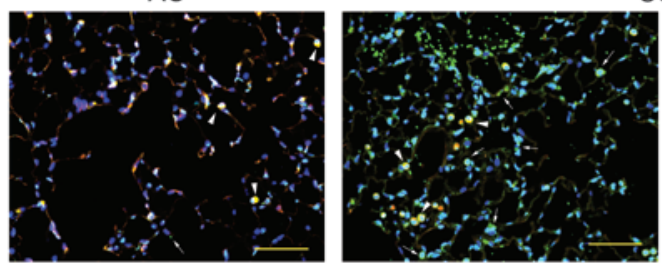

CS

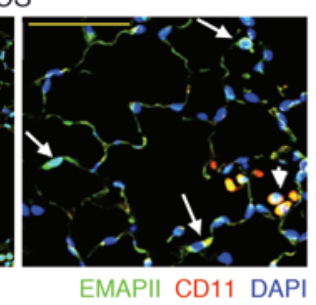

C

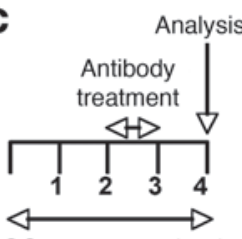

CS-exposure (mo)

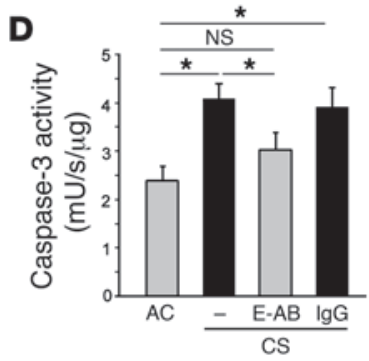

E

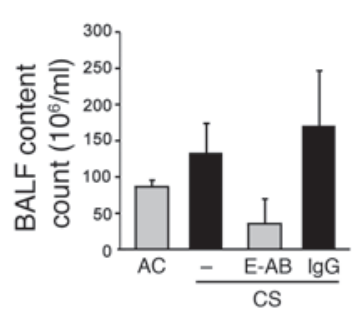

$\mathbf{F}$

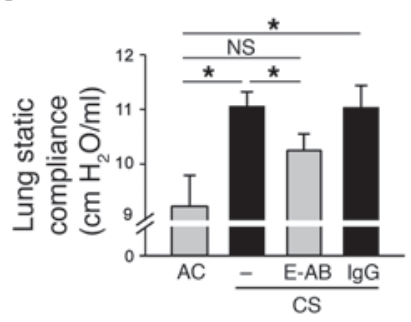

AC

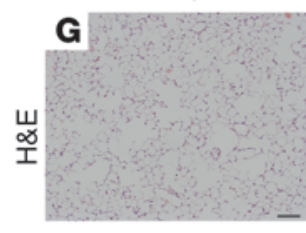

CS

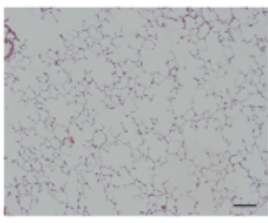

CS + EMAPII antibody $(E-A B)$

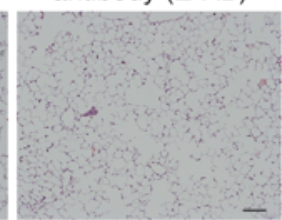

H

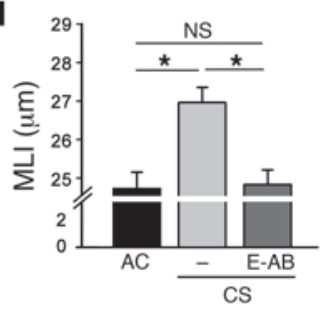

Figure 2

EMAPII is an essential mediator of CS-induced emphysema in mice. (A and B) EMAPII expression after CS exposure. (A) Production of EMAPII (pro and mature forms) in lung lysates in mice exposed to CS (4 weeks) compared with that in control mice exposed to ambient air (air control $[\mathrm{AC}]$ ), assessed by Western blot (mean densitometry units [DUs] normalized to vinculin $\pm \mathrm{SEM}$; ${ }^{*} P<0.05$ versus control; $n=5 /$ group). (B) EMAPII localization in the lung parenchyma detected by coimmunofluorescence with EMAPII antiserum (green), CD11b antibody (red), and DAPI (blue). In controls, EMAPII expression is sparse, and EMAPII colocalized with macrophages (arrowheads) (left panel). After CS exposure (2 months), EMAPII expression is increased both cellularly and extracellularly, less prominently colocalized with macrophages (arrowheads, middle), and increasingly expressed in parenchyma cells (arrows; right). Scale bar: $100 \mu \mathrm{m}$. (C-F) Effect of neutralizing EMAPII (E-AB) or control antibody (IgG) administered by nebulization $(50 \mu \mathrm{g} / 100 \mu \mathrm{l})$ during month 3 of CS exposure on CS-induced lung injury at 4 months. (C) Treatment protocol. (D) Apoptosis detected by caspase-3 activity in lung lysates (caspase units normalized by protein; mean $+\mathrm{SEM}$; ${ }^{*} P<0.05$, ANOVA). (E) Number of cells in BALF, and (F) lung static compliance (mean + SEM; ${ }^{\star} P<0.01$, ANOVA). (G) Representative H\&E-stained lung sections (scale bar: $100 \mu \mathrm{m}$ ) showing simplification of lung alveolar structures in response to CS but preserved alveolar architecture when treated with neutralizing EMAPII. (H) Morphometric measurement of MLIs (mean + SEM; * $P<0.05$, ANOVA; $n=5-12$ ).

upon apoptosis and caspase-3 activation and that increased EMAPII levels, either by i.t. recombinant protein delivery (Figure 3D) or by transgenic expression (Figure 1C), induce apoptosis in the lung.

To determine whether increased EMAPII expression would lead to further EMAPII induction in apoptosis-dependent fashion, we treated transgenic mature EMAPII-overexpressing mice with the caspase inhibitor Q-VD-OPH, administered via i.p. injection 3 times weekly for 8 weeks. Caspase inhibition markedly reduced EMAPII (proform) lung expression, as assessed by quantitative Western blot analysis of lung tissue in mature EMAPII-overexpressing DT mice (Figure 3E), indicating an apoptosis-dependent mechanism of amplified EMAPII protein production, even in the EMAPII transgenic mouse.

These results suggested that EMAPII upregulation and CS exposure may have synergistic effects on EMAPII release and apoptosis in the lung. To test this hypothesis, EMAPII-overexpressing
DT or ST control mice were first induced with doxycycline for 8 weeks and then exposed to CS for 4 weeks. EMAPII expression was increased in both non-smoking EMAPII-overexpressing DT mice and in cigarette smoking ST control mice, as detected by quantitative Western blot analysis in lung tissues (Figure 3F). Of note, when EMAPII-overexpressing DT mice were exposed to CS, a morethan-additive increase in mature EMAPII production was observed (Figure $3 \mathrm{~F}$ ), which was associated with a synergistic increase in the proapoptotic ceramide 16:0 in lung homogenates (Figure 3G).

Together, these data suggest that increased EMAPII expression is, at least in large part, linked to a feed-forward mechanism involving lung cell apoptosis and can be enhanced by CS exposure (schematic in Figure 3H).

EMAPII is elevated in active smokers and patients with COPD. To investigate the clinical relevance of our findings, EMAPII levels 
A

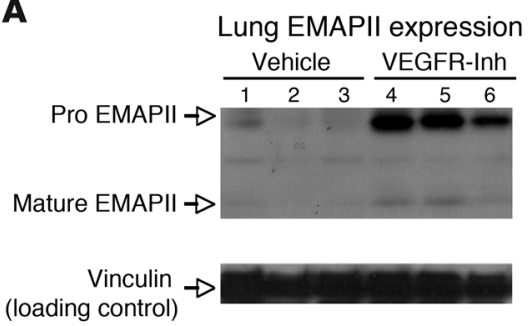

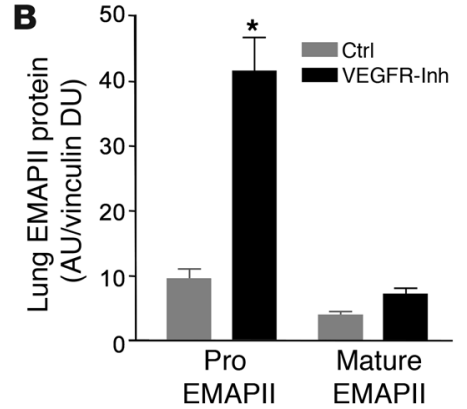

C
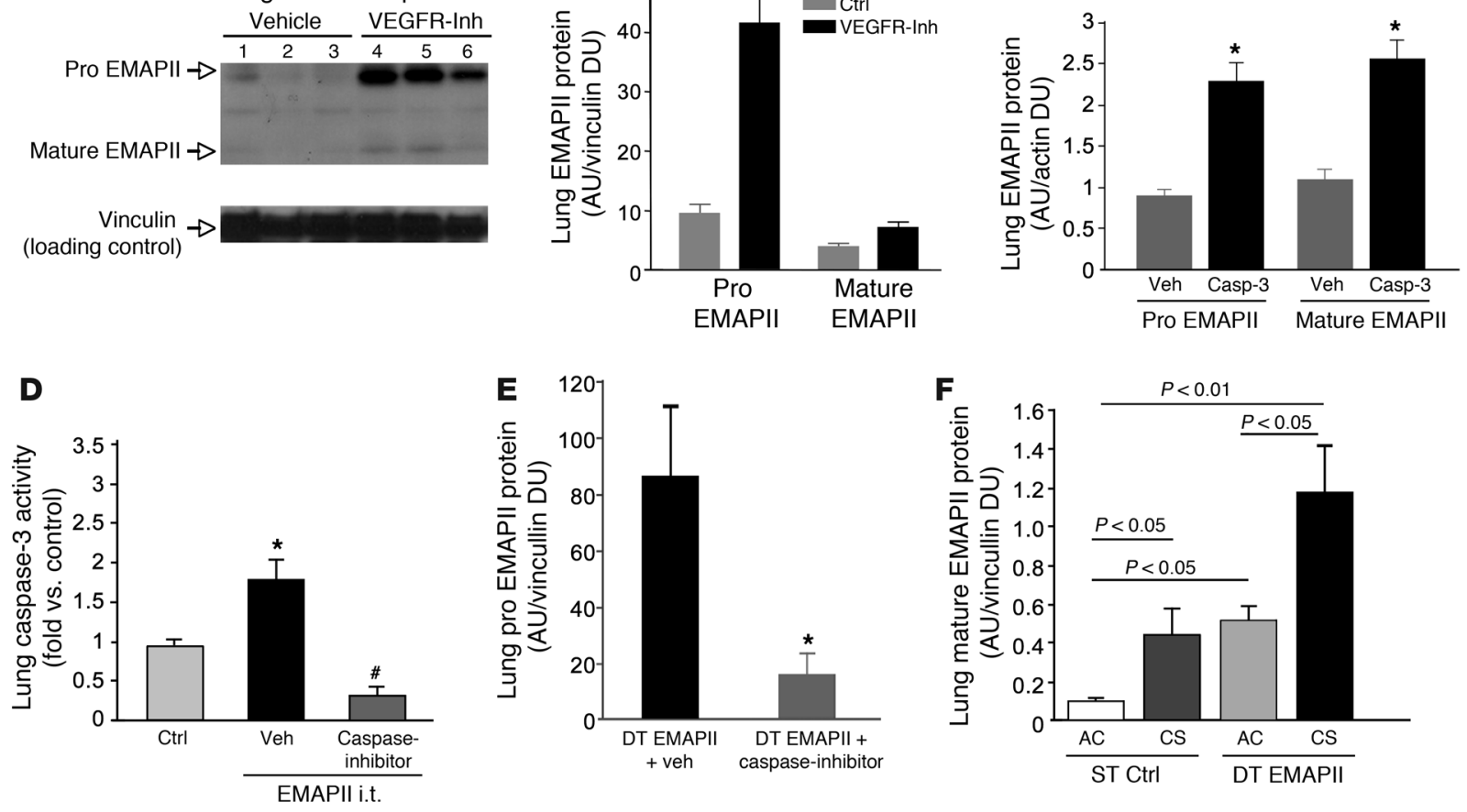

G

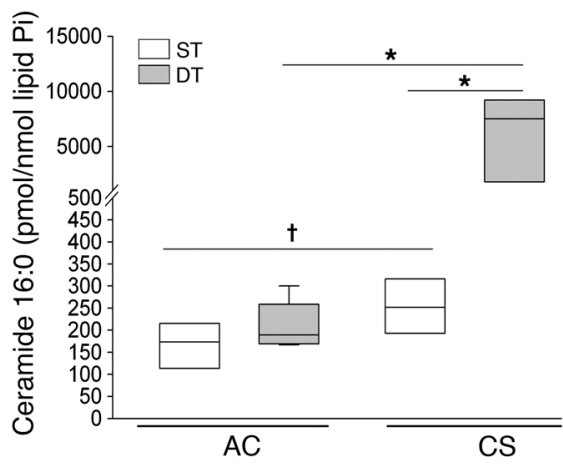

H

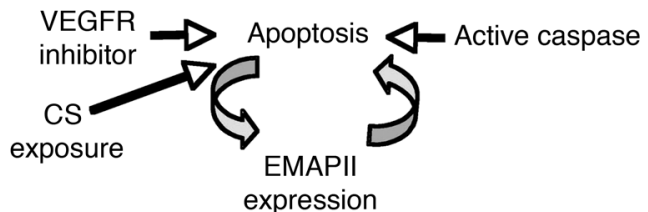

\section{Figure 3}

Apoptosis and EMAPII are mutually linked. (A) Immunoblot of lung EMAPII 4 weeks after VEGF receptor inhibitor treatment (VEGFR-inh, 20 $\mathrm{mg} / \mathrm{kg}$ ), (B) quantified as densitometry units normalized to vinculin (mean $\pm \mathrm{SEM}$; ${ }^{*} P<0.05$ versus control; $n=3 / \mathrm{group}$ ). Ctrl, control. (C) Lung EMAPII production following active caspase-3 (Casp-3) i.t. (0.8 U; 48 hours) or vehicle treatment (mean densitometry units \pm SEM; ${ }^{\star} P<0.05$ versus control; $n=4 /$ group). (D) Lung caspase-3 activity after recombinant mature EMAPII nebulization (EMAPII i.t.; $50 \mu \mathrm{g} / 100 \mu \mathrm{l} ; 2$ weeks) and caspase inhibitor (Q-VD-OPH, $25 \mu \mathrm{g} / 100 \mu \mathrm{l}$ i.p.) or vehicle (DMSO) treatment, relative to that of controls (saline i.t.). Mean $+\mathrm{SEM}$. ${ }^{\star} P<0.05$ versus control; $\# P<0.05$ versus EMAPII plus vehicle; $n=4$; ANOVA. (E) Lung EMAPII (proform) production in lung-specific mature EMAPIIoverexpressing mice (DT) treated with vehicle or caspase inhibitor 3 times weekly ( 8 weeks; mean $+\mathrm{SEM}$; ${ }^{*} P<0.05$ versus vehicle; $n=4 ; t$ test). $(\mathbf{F}$ and $\mathbf{G})$ Synergistic effect of EMAPII and CS on lung in EMAPII DT or control (ST) mice induced for 12 weeks, of which the last 4 weeks were combined with CS exposure. (F) EMAPII (mean densitometry units + SEM) and (G) ceramide 16:0 expression normalized by lipid phosphorus (Pi). ${ }^{*} P<0.01 ;{ }^{\dagger} P<0.05 ; n=4-5$. Lines within boxes show medians; bounds of boxes show 25 th and 75 th percentiles, respectively; and whiskers show 5th and 95th percentiles, respectively. (H) Schematic of apoptosis-anchored feed-forward loop of EMAPII production.

were determined in the acellular BALF of individuals who were actively smoking, without any known or diagnosed lung disease at the time of the evaluation, and were compared with levels in the BALF of healthy nonsmokers. EMAPII levels were quantified by densitometry of immunoblots of concentrated BALF with EMAPII-specific antibody normalized to protein loading by Ponceau S staining. Mature EMAPII was significantly $(P<0.01)$ increased in the BALF obtained from 9 actively smoking, healthy subjects in comparison with that of 10 nonsmokers (Figure 4A; the demographic details for these patients are shown in Supplemental Table 1). To further investigate whether the expression of EMAPII in human COPD persists even after cessation of smoking, we assessed EMAPII in 45 BALF samples obtained from a separate cohort of ex-smokers, with and without COPD, and compared these with those of healthy nonsmokers. Quantification, using immunoblot analysis of BAL supernatants, demonstrated that the 15 ex-smokers with COPD displayed significantly higher mature EMAPII levels compared with those of 15 healthy nonsmokers 
A

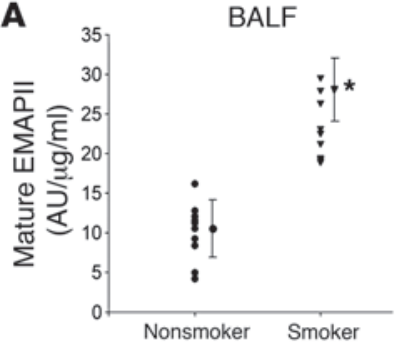

C EMAPII + DAPI (IF)
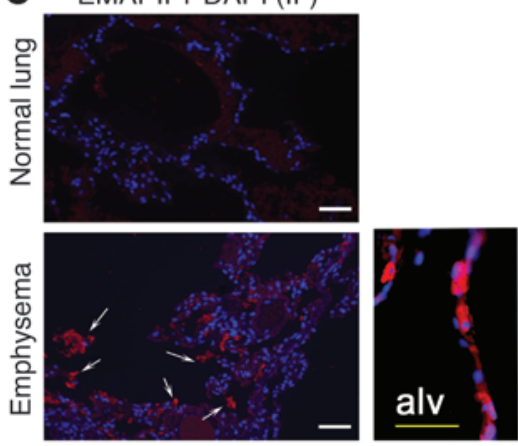

Human lung IHC

B

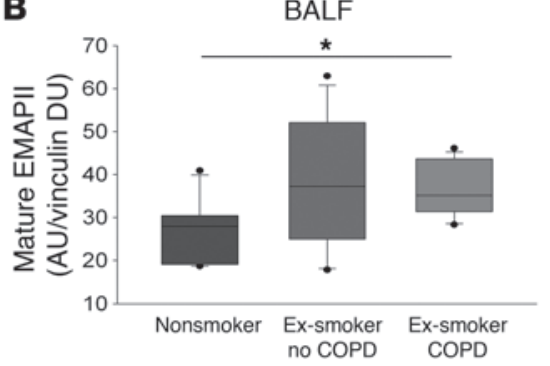

D

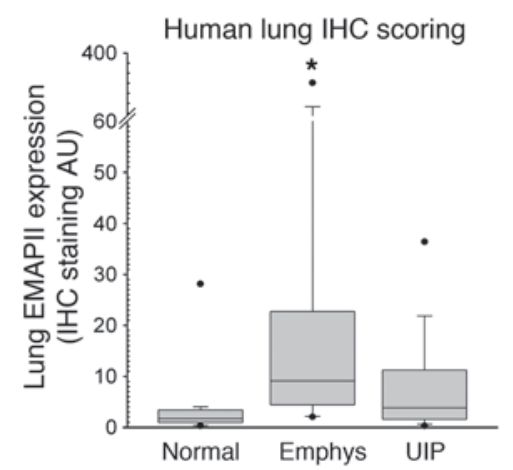

\section{Figure 4}

Increased EMAPII in human COPD. (A) Secreted EMAPII in the acellular BALF from otherwise healthy active smokers (triangles; $n=9$ ) compared with that from nonsmokers (circles; $n=10$ ). Mean arbitrary units measured by densitometry were normalized by protein concentration $\left(\mathrm{AU} / \mathrm{\mu g} / \mathrm{ml}\right.$; mean $\pm \mathrm{SD},{ }^{*} P=<0.01, t$ test). (B) Box plot of secreted mature EMAPII in acellular BALF from healthy nonsmokers, ex-smokers without COPD, or ex-smokers with COPD shown in arbitrary units normalized by vinculin densitometry units ( ${ }^{*} P<0.05$, ANOVA; $n=15$ /group). Demographic and clinical data are shown in Supplemental Table 1. (C) EMAPII detection by immunostaining with polyclonal EMAPII antibody (red, arrows; DAPI-nuclei are stained in blue) in lung parenchyma of patients with emphysema (bottom panels) compared with subjects without lung disease (normal lung, top panel). Note intense EMAPII staining in the alveolar wall (alv; inset) in emphysematous lungs. IF, immunofluorescence. Scale bar: $50 \mu \mathrm{m}$. (D) Box plot of lung EMAPII expression in lung sections from subjects without lung disease (normal; $n=4$ ), with emphysema (emphys; $n=9$ ), or with usual interstitial pneumonitis (UIP; $n=13$ ) (immunohistochemistry staining arbitrary units; * $P<0.05$ versus normal, ANOVA). (B and D) Lines within the boxes show medians; bounds of the boxes show 25th and the 75th percentiles of the data, respectively; and whiskers show outliers (5th and 95th percentiles, respectively).

(Figure 4B). We noted a high variability of EMAPII expression among the 15 ex-smokers who did not carry a diagnosis of COPD at the time of the BAL collection (the clinical details for these patients are shown in Supplemental Table 2). These findings were corroborated by a marked increase in EMAPII expression detected by immunohistochemistry of lung tissue obtained from patients with emphysema at the time of lung transplantation compared with healthy (normal) lung samples (Figure 4C). EMAPII staining was localized to alveolar walls and areas surrounding large bronchi. When quantified with automated blinded image analysis software, EMAPII expression was significantly increased in COPD lungs compared with normal lungs. We noted a wide range of EMAPII lung expression in the COPD lungs, whereas lung specimens with the usual interstitial pneumonitis form of pulmonary fibrosis did not exhibit increased EMAPII immunostaining compared with that of controls (Figure 4D).

\section{Discussion}

Targeting the proapoptotic protein EMAPII via antibody neutralization significantly reduced the development of CS-induced emphysema despite prior, concurrent, and subsequent exposure of the animals to CS. These findings, along with the demonstration that EMAPII overexpression in mice leads to further apoptosis-dependent increase in EMAPII production as part of a feed-forward loop, suggest a role of EMAPII in the perpetuation of lung injury induced by CS. This notion is also supported by our finding that high EMAPII levels in the BALF and lung parenchyma of individuals with COPD persist even after smoking has been ceased. In addition, the finding that mature EMAPII levels increased in smokers not (yet) diagnosed with COPD suggests the possibility that EMAPII may be upregulated even early in the time course of CSinduced lung injury. Studies using a larger number of patient specimens are needed to indicate whether EMAPII levels in the BALF or even plasma could be a biomarker of disease progression or severity. Our immunohistochemistry studies suggest that both lung epithelial cells and alveolar macrophages could be sources of EMAPII production in the mature lung, but the cell type responsible for the increased EMAPII measured in the BALF of patients with COPD remains to be determined.

The pathogenic role of EMAPII in emphysema was underscored by the finding that excessive EMAPII levels in the lungs of mice were sufficient to lead to emphysema-like morphological changes, apoptosis, and a monocytic inflammatory response in the lung, consistent with EMAPII's known proapoptotic and chemoattractive properties. Given that EMAPII is expressed in all cell types as a functional part of the tRNA-synthetase multienzyme complex $(9,11)$, EMAPII-deficient mice exposed to CS may exhibit a phenotype that extends beyond EMAPII chemokine-specific functions. We therefore elected to perform studies using EMAPII-neutralizing antibodies (28) that target only the secreted EMAPII. Treatment with these antibodies effectively inhibited airspace enlargement induced by CS exposure in a strain of mice that is highly susceptible to CS-induced emphysema. In the most commonly used animal model of emphysema, the magnitude of both morphometric and functional changes induced by CS, even in highly susceptible mouse strains such as the $\mathrm{DBA} / 2$ or the $\mathrm{C} 57 \mathrm{BL} / 6$, is of modest range of $20 \%-30 \%$ over a period of up to 6 months. We used the DBA/2 strain in our studies, because it has been reported to manifest an earlier onset of airspace enlargement (25). Morphometric and lung function assessments indicated emphysema development as early as 4 months following CS exposure, as compared with 6 months of exposure necessary to induce the disease in the C57BL/6 mouse strain. Compared to controls, similar magnitudes of airspace changes were observed upon lung-specific EMAPII overexpression for at least 3 months in developed (older than 3-month-old) mice. Of note, the baseline airspace size for these controls was in the same range as that reported for the CCSP-reverse tetracycline transactivator (CCSP- 
rtTA) transgenic mouse of C57BL/6 background (34). We decided to include the CCSP-rtTA background as control to account for any potential nonspecific effect of transgenic manipulation on airspaces (34) and to exclude doxycycline-specific effects, since both the ST (non-EMAPII-expressing) and DT (EMAPII-expressing) animals were similarly exposed to the drug. Interestingly, doxycycline could have even had an attenuating effect on airspace enlargement (35), which implies that the observed effects of EMAPII overexpression would have been even more prominent if an induction system other than doxycycline were to be used. Even with this transgenic approach, EMAPII overexpression induced both a more robust and a more rapid airspace enlargement compared with that triggered by CS exposure. Moreover, direct administration of recombinant EMAPII protein to the lungs led to a significant induction of apoptosis, being sufficient to enlarge airspaces as early as 4 weeks, in a dose-dependent manner.

EMAPII is known to be produced in response to oxidative or hypoxic stresses and through proteolytic cleavage from intracellular tRNA synthetase. Therefore, CS could upregulate EMAPII directly via oxidative stress or by proteolytic activation of caspases or other proteases. The increase in EMAPII in the lungs in response to active caspase- 3 instillation suggests that pro-EMAPII is released in response to apoptosis, while mature EMAPII secretion may result from further proteolytic processing of the proform. Interestingly, the mature EMAPII may block VEGF signaling (36) in the lung and thus could further contribute to apoptosis amplification and alveolar wall destruction induced by a VEGF-deprived state, previously linked to emphysema pathogenesis $(28,37)$. The increase in lung cell apoptosis was both attributable to EMAPII production and activity and was responsible for EMAPII secretion in the lung (schematic in Figure 3). This apoptosis-anchored autocrine loop may be a unique feature of the EMAPII signal amplification, distinct from other ligands of the chemokine receptor CXCR3, such as IP-10/ CXCL10, I-TAC/CXCL11, MIG/CXCL9, and PF4/CXCL4, some of which are also upregulated in the lungs or airways of patients with COPD (38-40). In addition, future studies will have to address the relative contribution of the monocyte chemoattractive function of EMAPII and subsequent activation of proteolysis to the development of airspace enlargement induced by this cytokine. Similar to our findings of a proapoptotic sphingolipid ceramide, which selfamplifies its synthesis via oxidative stress $(37,41)$, EMAPII may be the hub of a second important amplifying mechanism of emphysema development, one that occurs extracellularly and therefore may be both targetable by antibodies and measured as a biomarker in biological fluids.

To our knowledge, this is the first report of a neutralizing antibody-based treatment of lung emphysema. To avoid or minimize the potential development of anti-rat antibodies against the EMAPII antibodies, we limited the treatment duration to only 4 weeks. Future studies using murinized EMAPII antibody or studies in immune-tolerant mice will be able to extend the duration of antibody administration and to rule out antibody generation against the antibody treatment, thus potentially enhancing the efficacy of the biological therapy. In order to specifically neutralize lung EMAPII, the antibody was delivered by inhalation using a nebulizer, a method which previously demonstrated effective targeting of lung cytokines (27). However, this method required an increase in the antibody dose, given a considerable loss of approximately $20 \%-25 \%$ in the nebulizer (data not shown). Nevertheless, as we have demonstrated using labeled antibodies delivered by this tech- nique, there was significant antibody deposition in airways and alveolar spaces, with detectable antibody being recovered from plasma. Based on lung function and morphometric assessments, the EMAPII neutralization antibody was highly effective, even when only applied for a limited time period (during the third quarter of a 4-month CS exposure duration). Unlike the control IgG antibody, EMAPII neutralization ameliorated airspace enlargement and the remodeling of small but not of larger airways, which resulted in improved lung compliance by more than $30 \%$. The effect of EMAPII on alveolar space enlargement could be attributed to its apoptotic effect on endothelial cells, but the mechanism underlying its effect on small airway remodeling is less clear. Of note, similar changes induced by CS on the epithelial cells of small airways have been previously described and were attributed to a change in epithelial cell morphology, in particular, a decrease in the size of Clara cells (42). Future studies will determine whether EMAPII plays a role in this process.

Previous investigations conducted in vivo identified a physiological role for EMAPII in the maintenance of blood vessel integrity (4), control of aberrant vasculogenesis (43), and monocyte recruitment to apoptotic sites (24). Pathological consequences of EMAPII excess include the inhibition of neovascularization and airway epithelial morphogenesis in the developing lung (44-46). Our results add lung alveolar endothelial apoptosis and inflammation leading to emphysema-like disease as novel pathogenic roles for EMAPII. In addition, the positive feedback loop between EMAPII and apoptosis and the elevated levels of EMAPII in COPD suggest a role of EMAPII in the progression of lung disease in emphysema. Future investigations will have to determine whether circulating EMAPII may be useful as a noninvasive biomarker of disease activity. The remarkable effect of a relatively short treatment intervention with neutralizing antibodies on the lung function of mice exposed to CS suggests EMAPII could be a therapeutic target in this chronic obstructive lung disease.

\section{Methods}

Reagents and antibodies. All chemical reagents were purchased from SigmaAldrich, unless otherwise stated. EMAPII antiserum, used for immunoblotting and immunohistochemistry, was produced as recently described (24). Other antibodies used were from commercial sources, including Mac-3 (BioLegend), active caspase-3 (Cell Signaling), VE-Cadherin (BD Biosciences), and CD11b (Novus Biologicals).

Monoclonal anti-EMAPII antibody. The rat monoclonal neutralizing antibody M7/1 against mouse EMAPII was developed by immunizing Lewis rats with recombinant murine pro-EMAPII, as described previously (47). For purification of monoclonal antibodies for in vivo studies, hybridomas were grown in protein-free hybridoma medium (GIBCO-BRL), and antibodies were purified with protein G-Sepharose (Pharmacia).

Animal studies. Animal studies were approved by the Animal Care and Use Committee of Indiana University. DBA/2 mice were purchased from The Jackson Laboratory. C57BL/6 mice, used for EMAPII nebulization or inhaled antibody treatment, were from Harlan Laboratories.

EMAPII transgenic mice. We generated a lung-specific inducible EMAPII transgenic mouse by crossing the EMAPII responder mouse with homozygous transgenic mice containing the transactivator controlled by the lung epithelium-specific CCSP promoter (CCSP mouse line), provided by Jeffrey Whitsett (University of Cincinnati, Cincinnati, Ohio, USA) and Cong Yan (Indiana University). The EMAPII responder transgenic mouse contains the secreted (mature) form of EMAPII under a minimal promoter containing tetracycline-inducible sequences. Therefore, the murine mature 
EMAPII cloned from methylcholanthrene mouse tumor cells (12) and fused to a signal peptide derived from IFN- $\beta$ was inserted into the tet07-repeat containing plasmid pUD10-3 (provided by H. Bujard, ZMBH, Heidelberg, Germany) by using Sac II and Xho I insertion sites. The resulting plasmid was injected into oocytes for implantation into foster mice, and 3 transgenic lines were established out of 7 transgenic founders by backcrossing into C57BL/6. After crossing the resulting responder mice with the rtTA mice, we used for experiments the first generation mice, heterozygous for the EMAPII responder transgene and the CCSP transactivator with CCSP transactivator-only transgenic mice. Of note, only the double-transgene EMAPII/CCSP transactivator, but not the CCSP transactivator-only and the EMAPII-only transgenes, can induce EMAPII expression. With this design, CCSP transactivator background effects and tetracycline effects can be ruled out, as both groups can receive tetracycline. Transgenic mice were bred in our Association for Assessment and Accreditation of Laboratory Animal Care-accredited animal facility. DT EMAPII/CCSP-rtTA and ST CCSP-rtTA- or EMAPII-only mice were maintained on regular water until 3 to 4 months of age. Thereafter, the mice were placed on doxycycline treatment for up to 28 weeks. At the end of experiments, mice were euthanized, and the tissue was processed as described previously (37). The collection of BALF was performed by lavaging each animal's lungs 3 times with $0.6 \mathrm{ml}$ PBS, followed by BALF cell collection via centrifugation. BALF cells were counted and fixed as cytospin, followed by staining with Giemsa; the acellular fluid was snap frozen in liquid nitrogen and stored at $-80^{\circ} \mathrm{C}$ for further analysis.

Recombinant mature EMAPII administration and caspase inbibition. The fulllength and the mature forms of the EMAPII coding region were cloned into pPICZ A vector (Easy Select Pichia Expression Kit, Invitrogen), which contains combined His-and myc-tags at the $\mathrm{C}$ terminus. Expression of these 2 EMAPII forms was carried out according to the manufacturer's instructions and confirmed by SDS PAGE and Western blot analysis using rabbit polyclonal antibodies against EMAPII. For in vivo experiments, recombinant mature and pro-EMAPII protein from bacterial expression vectors (obtained as a gift of Marc Mirande, Laboratory of Structural Enzymology and Biochemistry, CNRS, Gif-sur-Yvette, France) was used. Purification of recombinant EMAPII was performed by 2 steps of affinity chromatography, using a Ni Sepharose 6 Fast Flow column (Life Sciences, GE), with intermediated anion exchange and desalting steps by gel filtration chromatography. These multiple purification steps were performed to reduce endotoxin contamination to less than $10 \mathrm{EU} / \mathrm{mg}$ protein. Mice (C57BL/6 mice; females, age 12 weeks; $n=5$ /group) were administered recombinant EMAPII protein $(10,50$, or $90 \mu \mathrm{g} / 100 \mu \mathrm{l})$ via aerosol nebulization via the nasal route, 3 times a week for the indicated time. Sterile PBS $(100 \mu \mathrm{l})$ was used as control. The caspase inhibitor Q-VD-OPH (MP Biologicals) was administered $(25 \mu \mathrm{g} / 100 \mu \mathrm{l}$ i.p.) 30 minutes prior to EMAPII nebulization and then 3 times a week for the duration of the experiment. DMSO $(2.5 \% / 100 \mu$ l i.p.) was used as vehicle control.

CS exposure. CS exposure was performed using a total body exposure method (48). Mice were exposed to CS or ambient air for up to 24 weeks. Briefly, mice were exposed to $11 \%$ mainstream and $89 \%$ sidestream smoke from reference cigarettes (Tobacco Research Institute) using the Teague $10 \mathrm{E}$ device. The exposure chamber atmosphere was monitored for total suspended particulates (average, $90 \mathrm{mg} / \mathrm{m}^{3}$ ) and carbon monoxide (average, $350 \mathrm{ppm}$ ). Two independent experiments were performed: in each, $\mathrm{DBA} / 2$ mice (female, age 12 weeks; $n=5-12 /$ group) were exposed to CS as described above or ambient air for 4 months; during the third month of CS exposure, the 2 groups of mice exposed to CS received either a monoclonal EMAPII-neutralizing antibody or isotype IgG control, and 1 group exposed to ambient air received isotype IgG control. Antibodies $(50 \mu \mathrm{g} / 100 \mu \mathrm{l}$ each inhalation) were administered via inhalation performed during the third month of smoking, 3 times a week, by exposing the nose of the mouse to the aerosol from a nebulizer unit with filler cap (Aerogen; 2.5-4.0 VMD). Mice were euthanized, and lungs were processed (37) on the day following the last of smoking exposure. In control experiments, Cy5-fluorescencelabeled antibodies or control nonlabeled IgG were administered once by nebulization, and lungs were harvested within 15 minutes for fluorescence microscopy. To further confirm parenchyma deposition, we investigated whether systemic absorption of antibody occurs. Biotinylated antibody (70 $\mu \mathrm{g} \mathrm{M7/1} \mathrm{IgG)} \mathrm{was} \mathrm{administered} \mathrm{by} \mathrm{inhalation} \mathrm{or} \mathrm{by} \mathrm{i.p.} \mathrm{injection} \mathrm{(as}$ control), followed by plasma collection and immunoadsorption on streptavidin-coated 96-well plates (Pierce), followed by antibody detection by HRP-conjugated anti-rat antibodies. Quantification was performed by using a standard curve of biotinylated IgG in mouse serum.

Active caspase-3 instillation. The i.t. administration of active caspase-3 was performed in mice by injection of $50 \mu \mathrm{l}$ of PBS solutions, containing either active caspase-3 (0.8 $\mu \mathrm{g}$; MBL) plus Chariot ( $1 \mu \mathrm{l}$; Active Motif) or Chariot $(1 \mu \mathrm{l})(30)$. The preparation of trachea, the i.t. delivery, and lung processing were performed as described previously (30).

Morphometric analysis. Morphometric analysis was performed on coded slides as described previously, using a macro developed by R.M. Tuder for MetaMorph (49). In addition, the point-sampled intercept method was used to determine the volume-weighted mean acinar airspace volume (31). Measurements were performed on coded, Richardson-stained sections of systematic, uniform, and random samples of lungs embedded into glycolmethacrylate.

Apoptosis. Apoptosis was detected in lysates or inflated fixed lung sections, enabling focus on alveoli, rather than large airways and vessels, via active caspase-3 immunostaining (Abcam and Cell Signaling) or in situ labeling of apoptotic DNA on murine lung, using rat serum as negative control (37). The immunostaining for active caspase- 3 was followed by DAPI (Molecular Probes) nuclear counterstaining. Executioner caspase (caspase-3 and/or caspase-7) activity was measured with the Apo-ONE Homogeneous Caspase-3/7 Assay Kit (Promega) as described previously (37). Human recombinant caspase-3 (Calbiochem) was used as positive control. Ceramide C16:0 was measured using combined liquid chromatography-tandem mass spectrometry of lipids extracted from lung homogenates and was normalized by total lipid phosphorus $\left(\mathrm{P}_{\mathrm{i}}\right)$ measurement as previously described, using ceramide 17:0 as internal standard (37).

$V E G F$ receptor blockade. VEGF receptor blockade was performed as previously described (37). Mice ( $n=4-6 /$ group) were injected with SU5416 (Calbiochem; $20 \mathrm{mg} / \mathrm{kg}$, subcutaneously) or vehicle (carboxymethylcellulose), and the mice were euthanized at 4 weeks.

Immunohistochemistry. Paraffin sections were blocked with $10 \%$ rabbit or goat serum and incubated with primary antibodies or control antibodies. Polyclonal anti-EMAPII (1:500), anti-caspase-3 (1:50; Cell Signaling), anti-Mac3 (1:50; BioLegend), or F4/80 (1:200) were incubated for 1 hour at room temperature or at $4{ }^{\circ} \mathrm{C}$ overnight. Bound antibody was detected using biotin-conjugated goat anti-rat IgG secondary antibody (1:100; Vector Laboratories) and streptavidin-coupled phycoerythrin or FITC (1:1,000; Vector Laboratories). For some applications (anti-Mac3 or anti-CD144; BD Biosciences) cryosections were used. Sections were counterstained with DAPI and mounted with Mowiol 488 (Calbiochem). Microscopy was performed on either a Nikon Eclipse (TE200S) inverted fluorescence or a combined confocal/multiphoton (Spectraphysics laser, Bio-Rad MRC1024MP) inverted system. Images were captured in a blinded fashion, and quantitative intensity (expression) data was obtained by MetaMorph Imaging software (Molecular Devices) as previously described (3).

Western blotting. Lung tissue was homogenized in RIPA buffer with protease inhibitors on ice, and Western blot analysis was performed as described previously (50). Briefly, proteins were separated by electrophoresis, blotted onto PVDF membranes, incubated with rabbit anti-EMAPII antiserum (SA 2847; 
1:1,000) and peroxidase-coupled goat anti-rabbit IgG (Dianova/Jackson ImmunoResearch Inc.) for 1 hour at room temperature, and developed using enhanced chemiluminescence (Amersham Pharmacia Biotech). The chemiluminescence signals were quantified by densitometry (ImageQuant) and normalized by housekeeping proteins (actin, GAPDH, or vinculin), which generated arbitrary units for each blot. These protein expression arbitrary units are therefore not comparable among various blots.

Lung compliance measurements. Lung compliance measurements were performed with the flexiVent system (Scireq). Mice were anesthetized with inhaled isoflurane in oxygen and orotracheally intubated with a 20-gauge intravenous cannula under direct vision. A good seal was confirmed by stable airway pressure during a sustained inflation. Isoflurane anesthesia was maintained throughout the measurements, and the mice were hyperventilated to eliminate spontaneous ventilation. Starting at FRC, the flexiVent was programmed to deliver 7 inspiratory volume steps, for a total volume of $1 \mathrm{ml}$, followed by 7 expiratory steps, pausing at each step for at least 1 second. Compliance was calculated by dividing the cumulative volume delivered at each step by the plateau pressure, averaging 3 pressure volumes loops for each mouse.

Cell cultures and detection of apoptosis by TUNEL. Human lung microvascular endothelial cells (Lonza) were grown at $37^{\circ} \mathrm{C}$ in a $95 \%$ humidified atmosphere of $5 \% \mathrm{v} / \mathrm{v} \mathrm{CO}_{2}$ in VascuLife EnGS Medium (Lifeline Cell Technology). About 20,000 cells were plated per well of 8-well Lab-Tek Chamber Slides (Nunc, Thermo Scientific), and apoptosis was induced with recombinant pro- and mature EMAPII. After 24 hours, cells were fixed in paraformaldehyde (4\%), and DNA strand breaks were assessed by TUNEL, using a fluorescein detection system according to the manufacturer's instructions (ApopTag Plus In Situ Apoptosis Fluorescein Detection Kit, Millipore). Sections were mounted in a solution of Biomeda mounting medium and analyzed with a Leitz fluorescence microscope. Quantification was performed by MetaMorph image software (50), and results were expressed by computing the ratios between TUNEL-positive and total (DAPI-positive) cells of triplicate measurements. As positive control, we treated cells with purified DNAase, and as negative control, we used slides for which the incubation with the transferase was omitted from the protocol.

Human specimens. Human specimens used for measurement of EMAPII expression were from various sources. Paraffin sections from fixed lungs were obtained from the Pathology Tissue Bank at the University of Colorado. Acellular BALF from patients with COPD who are ex-smokers and healthy nonsmoker controls was obtained under an IRB-approved protocol at the University of New York, Buffalo, New York, USA, and collected after the receipt of written informed consent from patients or their guardians. The de-identified use of these specimens for the current study was approved by the IRB at Indiana University. Acellular BALF from healthy nonsmokers and actively smoking individuals was obtained at Indiana University; the de-identified use of these specimens for the current study was approved by the IRB at Indiana University.

Statistics. Statistical analysis was performed with SigmaStat software, and ANOVA testing was followed by Student-Newman-Keuls post-hoc test or 2 -tailed Student's $t$ test. Statistical difference was accepted at $P<0.05$. Nonnormally distributed or large data sets were presented as box plots.

\section{Acknowledgments}

We are grateful to Jeffrey Whitsett and Cong Yan for providing us with the CCSP-rtTA transactivator mouse line. We thank Jana Garrison, Jeremy J. Adamowicz, Patricia Smith, Osato J. Ogbeifun, Nagesh Gollahalli, Yong Gao, and Abby Renwick for technical support and Joud Hajjar for assistance with image capture for lung morphometry. This work was supported by NIH-NHLBI contract grant number R01 HL090950 (to M. Clauss and I. Petrache); parts of this work were also sponsored by NIH-NHLBI contract grant number R01 HL 077328 (to I. Petrache), DFG grant SFB 547-C5 (to M. Clauss), Cluster of Excellence EXC 306 (to H. Fehrenbach); and European Commission grant LSHM-CT2005-018725 (to R. Voswinckel).

Received for publication May 31, 2010, and accepted in revised form March 30, 2011.

Address correspondence to: Irina Petrache, Indiana University, Division of Pulmonary, Allergy, Critical Care and Occupational Medicine, Walther Hall R3, C400, 980 W. Walnut Street, Indianapolis, Indiana 46202, USA. Phone: 317.278.2894; Fax: 317.278.7030; E-mail: ipetrach@iupui.edu.
1. Tuder RM, Petrache I, Elias JA, Voelkel NF, Henson PM. Apoptosis and emphysema: the missing link. Am J Respir Cell Mol Biol. 2003;28(5):551-554.

2. Park JW, Ryter SW, Choi AM. Functional significance of apoptosis in chronic obstructive pulmonary disease. Copd. 2007;4(4):347-353.

3. Giordano RJ, et al. Targeted induction of lung endothelial cell apoptosis causes emphysemalike changes in the mouse. J Biol Chem. 2008; 283(43):29447-29460.

4. Kao J, et al. Endothelial monocyte-activating polypeptide II. A novel tumor-derived polypeptide that activates host-response mechanisms. J Biol Chem. 1992;267(28):20239-20247.

5. Kao J, et al. Characterization of a novel tumor-derived cytokine. Endothelial- monocyte activating polypeptide II. J Biol Chem. 1994;269(40):25106-25119.

6. Schwarz MA, et al. Endothelial-monocyte activating polypeptide II, a novel antitumor cytokine that suppresses primary and metastatic tumor growth and induces apoptosis in growing endothelial cells. J Exp Med. 1999;190(3):341-354.

7. Berger AC, et al. Endothelial monocyte activating polypeptide II induces endothelial cell apoptosis and may inhibit tumor angiogenesis. Microvasc Res. 2000;60(1):70-80.

8. Hou Y, et al. Endothelial-monocyte-activating polypeptide II induces migration of endothelial progenitor cells via the chemokine receptor CXCR3.
Exp Hematol. 2006;34(8):1125-1132.

9. Wakasugi K, Schimmel P. Highly differentiated motifs responsible for two cytokine activities of a split human tRNA synthetase. J Biol Chem. 1999; 274(33):23155-23159.

10. Kao J, et al. A peptide derived from the amino terminus of endothelial-monocyte-activating polypeptide II modulates mononuclear and polymorphonuclear leukocyte functions, defines an apparently novel cellular interaction site, and induces an acute inflammatory response. J Biol Chem. 1994;269(13):9774-9782.

11. Quevillon S, Agou F, Robinson JC, Mirande M. The p43 component of the mammalian multisynthetase complex is likely to be the precursor of the endothelial monocyte-activating polypeptide II cytokine. J Biol Chem. 1997;272(51):32573-32579.

12. Knies UE, et al. Regulation of endothelial monocyte-activating polypeptide II release by apoptosis. Proc Natl Acad Sci U S A. 1998;95(21):12322-12327.

13. Barnett G, Jakobsen AM, Tas M, Rice K, Carmichael J, Murray JC. Prostate adenocarcinoma cells release the novel proinflammatory polypeptide EMAP-II in response to stress. Cancer Res. 2000; 60(11):2850-2857.

14. Matschurat S, et al. Regulation of EMAP II by Hypoxia. Am J Pathol. 2003;162(1):93-103.

15. Journeay WS, Janardhan KS, Singh B. Expression and function of endothelial monocyte-activat- ing polypeptide-II in acute lung inflammation. Inflamm Res. 2007;56(5):175-181.

16. Behrensdorf HA, van de Craen M, Knies UE, Vandenabeele $\mathrm{P}$, Clauss $\mathrm{M}$. The endothelial monocyteactivating polypeptide II (EMAP II) is a substrate for caspase-7. FEBS Lett. 2000;466(1):143-147.

17. Wakasugi K, Schimmel P. Two distinct cytokines released from a human aminoacyl-tRNA synthetase. Science. 1999;284(5411):147-151.

18. Zhang FR, Schwarz MA. Pro-EMAP II is not primarily cleaved by caspase-3 and -7. Am J Physiol Lung Cell Mol Physiol. 2002;282(6):L1239-L1244.

19. Liu J, Schwarz MA. Identification of protease-sensitive sites in human endothelial-monocyte activating polypeptide II protein. Exp Cell Res. 2006; 312(12):2231-2237.

20. Shalak V, Kaminska M, Mitnacht-Kraus R, Vandenabeele P, Clauss M, Mirande M. The EMAPII cytokine is released from the mammalian multisynthetase complex after cleavage of its $\mathrm{p} 43$ /proEMAPII component. J Biol Chem. 2001;276(26):23769-23776.

21. Shapiro SD. Proteinases in chronic obstructive pulmonary disease. Biochem Soc Trans. 2002; 30(2):98-102.

22. Clark JC, et al. FGF-10 disrupts lung morphogenesis and causes pulmonary adenomas in vivo. Am J Physiol Lung Cell Mol Physiol. 2001;280(4):L705-L715.

23. Qu P, et al. Stat3 downstream genes serve as biomarkers in human lung carcinomas and chronic 
obstructive pulmonary disease. Lung Cancer. 2009; 63(3):341-347.

24. Knies UE, Kroger S, Clauss M. Expression of EMAP II in the developing and adult mouse. Apoptosis. 2000;5(2):141-151.

25. Bartalesi B, et al. Different lung responses to cigarette smoke in two strains of mice sensitive to oxidants. Eur Respir J. 2005;25(1):15-22.

26. Rajashekhar G, et al. A monoclonal rat anti-mouse EMAP II antibody that functionally neutralizes pro- and mature-EMAP II in vitro. J Immunol Methods. 2009;350(1-2):22-28.

27. Shen HH, Xu F, Zhang GS, Wang SB, Xu WH. CCR3 monoclonal antibody inhibits airway eosinophilic inflammation and mucus overproduction in a mouse model of asthma. Acta Pharmacol Sin. 2006; 27(12):1594-1599.

28. Kasahara Y, et al. Inhibition of VEGF receptors causes lung cell apoptosis and emphysema. J Clin Invest. 2000;106(11):1311-1319.

29. Aoshiba K, Yokohori N, Nagai A. Alveolar wall apoptosis causes lung destruction and emphysematous changes. Am J Respir Cell Mol Biol. 2003; 28(5):555-562.

30. Petrache I, et al. \{alpha\}-1 Antitrypsin Inhibits Caspase-3 Activity, Preventing Lung Endothelial Cell Apoptosis. Am J Pathol. 2006;169(4):1155-1166.

31. Fehrenbach A, et al. Stereological estimation of the volume weighted mean volumes of alveoli and acinar pathways in the rat lung to characterise alterations after ischaemia/reperfusion. J Anat. 1999; 194(pt 1):127-135.

32. Knudsen L, Weibel ER, Gundersen HJ, Weinstein FV, Ochs M. Assessment of air space size characteristics by intercept (chord) measurement: an accu- rate and efficient stereological approach. J Appl Physiol. 2010;108(2):412-421.

33. Caserta TM, Smith AN, Gultice AD, Reedy MA, Brown TL. Q-VD-OPh, a broad spectrum caspase inhibitor with potent antiapoptotic properties. Apoptosis. 2003;8(4):345-352.

34. Sisson TH, et al. Expression of the reverse tetracyclinetransactivator gene causes emphysema-like changes in mice. Am J Respir Cell Mol Biol. 2006;34(5):552-560.

35. Rossiter HB, Scadeng M, Tang K, Wagner PD, Breen EC. Doxycycline treatment prevents alveolar destruction in VEGF-deficient mouse lung. $J$ Cell Biochem. 2008;104(2):525-535.

36. Awasthi N, Schwarz MA, Verma V, Cappiello C, Schwarz RE. Endothelial monocyte activating polypeptide II interferes with VEGF-induced proangiogenic signaling. Lab Invest. 2009;89(1):38-46.

37. Petrache I, et al. Ceramide upregulation causes pulmonary cell apoptosis and emphysema-like disease in mice. Nat Med. 2005;11(5):491-498.

38. Grumelli S, et al. An immune basis for lung parenchymal destruction in chronic obstructive pulmonary disease and emphysema. PLoS Med. 2004;1(1):e8.

39 . Saetta M, et al. Increased expression of the chemokine receptor CXCR3 and its ligand CXCL10 in peripheral airways of smokers with chronic obstructive pulmonary disease. Am J Respir Crit Care Med. 2002;165(10):1404-1409.

40. Costa C, Rufino R, Traves SL, Lapa ESJR, Barnes PJ, Donnelly LE. CXCR3 and CCR5 chemokines in induced sputum from patients with COPD. Chest. 2008;133(1):26-33.

41. Petrache I, et al. Superoxide dismutase protects against apoptosis and alveolar enlargement induced by ceramide. Am J Physiol Lung Cell Mol Physiol. 2008;
295(1):L44-L53.

42. Adair-Kirk TL, et al. Distal airways in mice exposed to cigarette smoke: Nrf2-regulated genes are increased in Clara cells. Am J Respir Cell Mol Biol. 2008; 39(4):400-411.

43. Marvin MR, et al. A novel tumor-derived mediator that sensitizes cytokine-resistant tumors to tumor necrosis factor. J Surg Res. 1996;63(1):248-255.

44. Schwarz MA, Zhang F, Gebb S, Starnes V, Warburton D. Endothelial monocyte activating polypeptide II inhibits lung neovascularization and airway epithelial morphogenesis. Mech Dev. 2000;95(1-2):123-132.

45. Quintos-Alagheband ML, White CW, Schwarz MA. Potential role for antiangiogenic proteins in the evolution of bronchopulmonary dysplasia. Antioxid Redox Signal. 2004;6(1):137-145.

46. Warburton D, Schwarz M, Tefft D, Flores-Delgado G, Anderson KD, Cardoso WV. The molecular basis of lung morphogenesis. Mech Dev. 2000;92(1):55-81.

47. Rajashekhar $\mathrm{G}$, et al. A monoclonal rat anti-mouse EMAP II antibody that functionally neutralizes pro- and mature-EMAP II in vitro. J Immunol Methods. 2009;350(1-2):22-28.

48. Cavarra E, et al. Effects of cigarette smoke in mice with different levels of alpha(1)-proteinase inhibitor and sensitivity to oxidants. Am J Respir Crit Care Med. 2001;164(5):886-890.

49. Tuder RM, et al. Oxidative stress and apoptosis interact and cause emphysema due to vascular endothelial growth factor receptor blockade. Am J Respir Cell Mol Biol. 2003;29(1):88-97.

50. Castro CM, Yang Y, Zhang Z, Linnoila RI. Attenuation of pulmonary neuroendocrine differentiation in mice lacking Clara cell secretory protein. Lab Invest. 2000;80(10):1533-1540. 